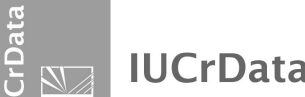

ISSN 2414-3146

Received 20 September 2016

Accepted 2 October 2016

Edited by $\mathrm{H}$. Stoeckli-Evans, University of Neuchâtel, Switzerland

Keywords: crystal structure; quinoline; carbohydrazide; hydrogen bonding.

CCDC reference: 1507748

Structural data: full structural data are available from iucrdata.iucr.org

\section{(E)-N'-Benzylidene-2-phenylquinoline-4-carbo- hydrazide}

Joel T. Mague, ${ }^{a}$ Shaaban K. Mohamed, ${ }^{b, c}$ Mehmet Akkurt, ${ }^{\text {d }}$ Mustafa R. Albayati and Ehab A. Ahmed ${ }^{\mathrm{f}}$ *

${ }^{\mathbf{a}}$ Department of Chemistry, Tulane University, New Orleans, LA 70118, USA, ${ }^{\mathbf{b}}$ Chemistry and Environmental Division, Manchester Metropolitan University, Manchester M1 5GD, England, ' ${ }^{\mathbf{C}}$ Chemistry Department, Faculty of Science, Minia University, 61519 El-Minia, Egypt, dDepartment of Physics, Faculty of Sciences, Erciyes University, 38039 Kayseri, Turkey, ${ }^{\mathbf{e}}$ Kirkuk University, College of Education, Department of Chemistry, Kirkuk, Iraq, and 'Department of Chemistry, College of Education, Tikrit University, Iraq. *Correspondence e-mail: shaabankamel@yahoo.com

In the title compound, $\mathrm{C}_{23} \mathrm{H}_{17} \mathrm{~N}_{3} \mathrm{O}$, there is a short intramolecular $\mathrm{C}-\mathrm{H} \cdots \mathrm{O}$ contact present, and the conformation about the $\mathrm{C}=\mathrm{N}$ bond is $E$. The phenyl and benzylidene rings make dihedral angles of 28.21 (15) and $37.65(14)^{\circ}$ with the mean plane of the quinoline moiety. In the crystal, molecules are linked by $\mathrm{N}-\mathrm{H} \cdots \mathrm{O}$ and $\mathrm{C}-\mathrm{H} \cdots \mathrm{O}$ hydrogen bonds, forming chains propagating along [001], with the $\mathrm{O}$ atom accepting three hydrogen bonds.
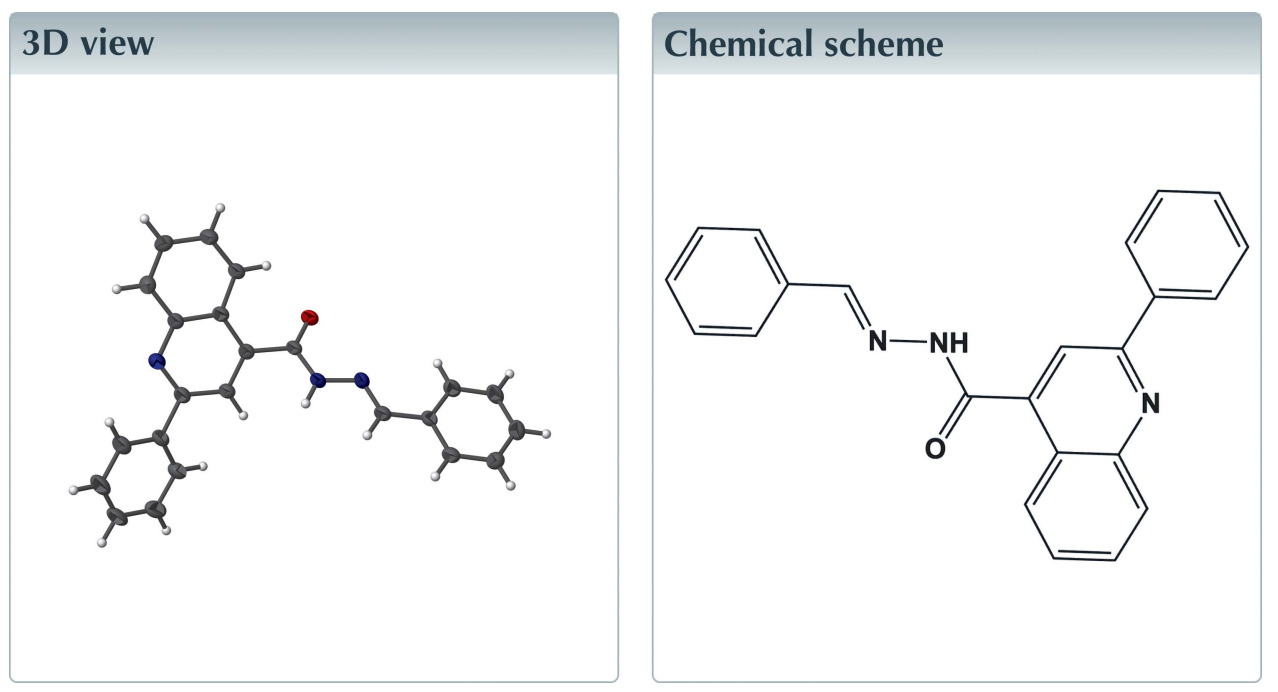

\section{Structure description}

It has been reported that quinoline derivatives serve as antagonists (Bennacef et al., 2007), analgesic agents (Gopalsamy \& Pallai, 1997), 5HT3 antagonists (Anzini et al., 1995) and structural subunits of natural products (Sivaprasad et al., 2006). Among the compounds of a quinoline series, Atophan and its derivatives have shown a variety of biological effects (Muscia et al., 2008; Wang et al., 2009). Moreover, hydrazide-hydrazone compounds are found to be associated with various biological activities such as antimicrobial, anticonvulsant, analgesic, anti-inflammatory, anti-platelet, anti-tubercular and anti-tumour properties (Mohamed et al., 2015). As part of our studies in this area, we now report the crystal structure of the title compound.

The molecular structure of the title compound is illustrated in Fig. 1. The quinoline moiety is slightly twisted as indicated by the dihedral angle of $2.27(16)^{\circ}$ between the $\mathrm{C} 1-$ $\mathrm{C} 6$ and N1/C6-C9 rings. The phenyl (C10-C15) and benzylidene (C18-C23) rings make dihedral angles of $28.21(15)$ and $37.65(14)^{\circ}$, respectively, with the mean plane of the 


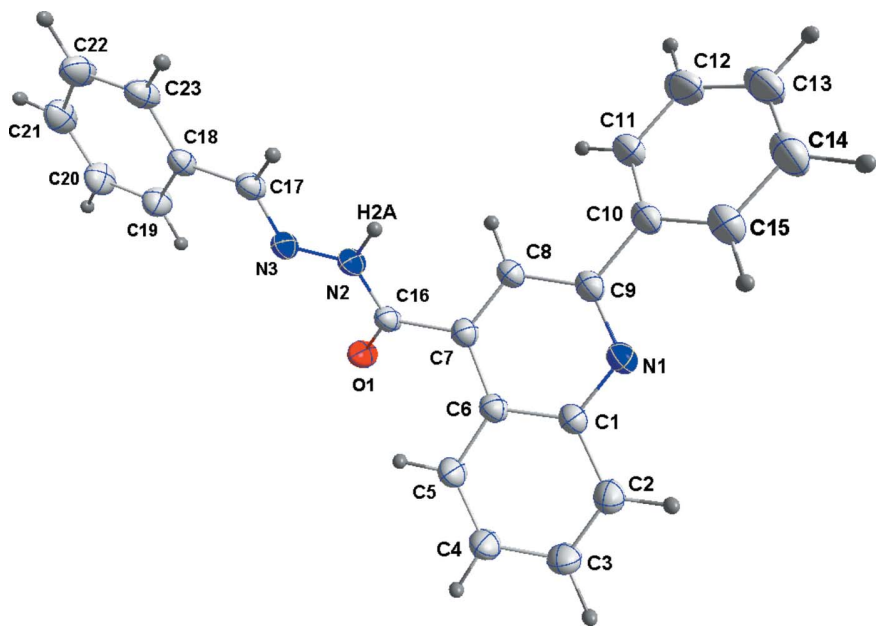

Figure 1

The molecular structure of the title compound, showing the atom labelling and $50 \%$ probability displacement ellipsoids.

quinoline moiety. The conformation about the $\mathrm{C} 17=\mathrm{N} 3$ bond is $E$ and there is a short $\mathrm{C} 5-\mathrm{H} 5 \cdots \mathrm{O} 1$ contact in the molecule (Table 1).

In the crystal, $\mathrm{N} 2-\mathrm{H} 2 A \cdots \mathrm{O} 1^{\mathrm{i}}, \mathrm{C} 8-\mathrm{H} 8 \cdots \mathrm{O} 1^{\mathrm{i}}$ and $\mathrm{C} 17-$ H17. . O $1^{\mathrm{i}}$ [symmetry code: (i) $x,-y+1, z-\frac{1}{2}$ ] hydrogen bonds link the molecules into chains along the $c$-axis direction (Table 1 and Fig. 2).

\section{Synthesis and crystallization}

The title compound was prepared in $89 \%$ yield according to a reported procedure (Mohamed et al., 2016). Colourless needle-like crystals of the title compound were obtained by recrystallization from ethanol solution (m.p. 515-518 K).

\section{Refinement}

Crystal and refinement details are given in Table 2.

\section{Acknowledgements}

The support of NSF-MRI Grant No. 1228232 for the purchase of the diffractometer and Tulane University for support of the

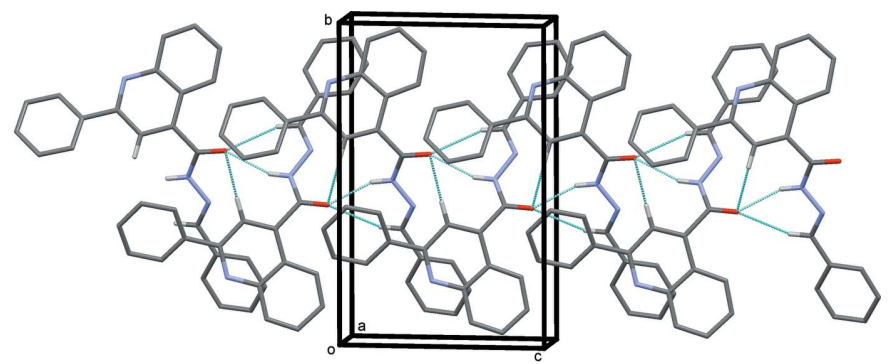

Figure 2

A view along the $a$ axis of the crystal packing of the title compound. The $\mathrm{N}-\mathrm{H} \cdots \mathrm{O}$ and $\mathrm{C}-\mathrm{H} \cdots \mathrm{O}$ hydrogen bonds are shown as dashed lines (see Table 1) and, for clarity, only $\mathrm{H}$ atoms $\mathrm{H} 26, \mathrm{H} 8$ and $\mathrm{H} 17$ have been included.
Table 1

Hydrogen-bond geometry $\left(\AA,^{\circ}\right)$.

\begin{tabular}{lllll}
\hline$D-\mathrm{H} \cdots A$ & $D-\mathrm{H}$ & $\mathrm{H} \cdots A$ & $D \cdots A$ & $D-\mathrm{H} \cdots A$ \\
\hline $\mathrm{C} 5-\mathrm{H} 5 \cdots \mathrm{O} 1$ & 0.95 & 2.34 & $2.939(3)$ & 121 \\
$\mathrm{~N} 2-\mathrm{H} 2 A \cdots \mathrm{O} 1^{\mathrm{i}}$ & $0.88(4)$ & $2.08(4)$ & $2.907(3)$ & $155(4)$ \\
$\mathrm{C} 8-\mathrm{H} 8 \cdots \mathrm{O} 1^{\mathrm{i}}$ & 0.95 & 2.50 & $3.288(3)$ & 140 \\
$\mathrm{C} 17-\mathrm{H} 17 \cdots \mathrm{O} 1^{\mathrm{i}}$ & 0.95 & 2.52 & $3.268(3)$ & 136 \\
\hline
\end{tabular}

Symmetry code: (i) $x,-y+1, z-\frac{1}{2}$.

Table 2

Experimental details.

Crystal data

Chemical formula

$M_{\mathrm{r}}$

Crystal system, space group

Temperature (K)

$a, b, c(\AA)$

$\beta\left({ }^{\circ}\right)$

$V\left(\AA^{3}\right)$

$Z$

Radiation type

$\mu\left(\mathrm{mm}^{-1}\right)$

Crystal size (mm)

$\mathrm{C}_{23} \mathrm{H}_{17} \mathrm{~N}_{3} \mathrm{O}$

351.39

Monoclinic, $P c$

150

7.6704 (8), 13.2898 (13), 9.0627 (9)

$107.220(6)$

$882.42(16)$

2

$\mathrm{Cu} K \alpha$

0.66

$0.24 \times 0.07 \times 0.04$

Data collection

Diffractometer

Absorption correction

$T_{\min }, T_{\max }$

No. of measured, independent and observed $[I>2 \sigma(I)]$ reflections

$R_{\text {int }}$

$(\sin \theta / \lambda)_{\max }\left(\AA^{-1}\right)$

Bruker D8 VENTURE PHOTON
100 CMOS
Multi-scan ( $A A D A B S$; Bruker,
$2016)$
$0.83,0.97$
$6668,2637,2394$
0.037
0.618

$0.037,0.087,1.07$
2637
250
2
H atoms treated by a mixture of
$\quad$ independent and constrained
refinement
$0.16,-0.16$

$\Delta \rho_{\max }, \Delta \rho_{\min }\left(\mathrm{e} \AA^{-3}\right)$ refinement

Refinement

$R\left[F^{2}>2 \sigma\left(F^{2}\right)\right], w R\left(F^{2}\right), S$

No. of reflections

No. of parameters

No. of restraints

$\mathrm{H}$-atom treatment

Computer programs: APEX3 and SAINT (Bruker, 2016), SHELXT (Sheldrick, 2015a), SHELXL2014 (Sheldrick, 2015b), DIAMOND (Brandenburg \& Putz, 2012), Mercury (Macrae et al., 2008) and SHELXTL (Sheldrick, 2008).

Tulane Crystallography Laboratory are gratefully acknowledged.

\section{References}

Anzini, M., Cappelli, A., Vomero, S., Giorgi, G., Langer, T., Hamon, M., Merahi, N., Emerit, B. M., Cagnotto, A., Skorupska, M., Mennini, T. \& Pinto, J. C. (1995). J. Med. Chem. 38, 2692-2704.

Bennacef, I., Perrio, C., Lasne, M. C. \& Barré, L. (2007). J. Org. Chem. 72, 2161-2165.

Brandenburg, K. \& Putz, H. (2012). DIAMOND. Crystal Impact GbR, Bonn, Germany.

Bruker (2016). APEX3, SAINT and SADABS. Bruker AXS Inc., Madison, Wisconsin, USA.

Gopalsamy, A. \& Pallai, P. V. (1997). Tetrahedron Lett. 38, 907-910. Macrae, C. F., Bruno, I. J., Chisholm, J. A., Edgington, P. R., McCabe, P., Pidcock, E., Rodriguez-Monge, L., Taylor, R., van de Streek, J. \& Wood, P. A. (2008). J. Appl. Cryst. 41, 466-470.

Mohamed, S. K., Mague, J. T., Akkurt, M., Mohamed, A. F. \& Albayati, M. R. (2015). Acta Cryst. E71, o957-o958. 


\section{data reports}

Mohamed, S. K., Mague, J. T., Akkurt, M., Mohamed, A. F. \& Albayati, M. R. (2016). IUCrData, 1, x160662.

Muscia, G. C., Carnevale, J. P., Bollini, M. \& Asís, S. E. (2008). J. Heterocycl. Chem. 45, 611-614.

Sheldrick, G. M. (2008). Acta Cryst. A64, 112-122.

Sheldrick, G. M. (2015a). Acta Cryst. A71, 3-8.
Sheldrick, G. M. (2015b). Acta Cryst. C71, 3-8.

Sivaprasad, G., Rajesh, R. \& Perumal, P. T. (2006). Tetrahedron Lett. 47, 1783-1785.

Wang, L.-M., Hu, L., Chen, H.-J., Sui, Y.-Y. \& Shen, W. (2009). J. Fluorine Chem. 130, 406-409. 


\section{full crystallographic data}

IUCrData (2016). 1, x161544 [https://doi.org/10.1107/S2414314616015443]

\section{$(E)-N^{\prime}$-Benzylidene-2-phenylquinoline-4-carbohydrazide}

Joel T. Mague, Shaaban K. Mohamed, Mehmet Akkurt, Mustafa R. Albayati and Ehab A. Ahmed

(E)-N'-Benzylidene-2-phenylquinoline-4-carbohydrazide

Crystal data

$\mathrm{C}_{23} \mathrm{H}_{17} \mathrm{~N}_{3} \mathrm{O}$

$M_{r}=351.39$

Monoclinic, $P c$

$a=7.6704(8) \AA$

$b=13.2898(13) \AA$

$c=9.0627(9) \AA$

$\beta=107.220(6)^{\circ}$

$V=882.42(16) \AA^{3}$

$Z=2$

\section{Data collection}

Bruker D8 VENTURE PHOTON 100 CMOS diffractometer

Radiation source: INCOATEC I $\mu \mathrm{S}$ micro-focus source

Mirror monochromator

Detector resolution: 10.4167 pixels $\mathrm{mm}^{-1}$

$\omega$ scans

Absorption correction: multi-scan

(SADABS; Bruker, 2016)

\section{Refinement}

Refinement on $F^{2}$

Least-squares matrix: full

$R\left[F^{2}>2 \sigma\left(F^{2}\right)\right]=0.037$

$w R\left(F^{2}\right)=0.087$

$S=1.07$

2637 reflections

250 parameters

2 restraints

Primary atom site location: structure-invariant direct methods

Secondary atom site location: difference Fourier map

Hydrogen site location: mixed
$F(000)=368$

$D_{\mathrm{x}}=1.323 \mathrm{Mg} \mathrm{m}^{-3}$

$\mathrm{Cu} K \alpha$ radiation, $\lambda=1.54178 \AA$

Cell parameters from 5448 reflections

$\theta=3.3-72.3^{\circ}$

$\mu=0.66 \mathrm{~mm}^{-1}$

$T=150 \mathrm{~K}$

Needles, colourless

$0.24 \times 0.07 \times 0.04 \mathrm{~mm}$

$T_{\min }=0.83, T_{\max }=0.97$

6668 measured reflections

2637 independent reflections

2394 reflections with $I>2 \sigma(I)$

$R_{\text {int }}=0.037$

$\theta_{\max }=72.3^{\circ}, \theta_{\min }=3.3^{\circ}$

$h=-9 \rightarrow 8$

$k=-16 \rightarrow 15$

$l=-10 \rightarrow 11$

$\mathrm{H}$ atoms treated by a mixture of independent and constrained refinement

$w=1 /\left[\sigma^{2}\left(F_{\mathrm{o}}^{2}\right)+(0.0333 P)^{2}+0.1673 P\right]$

where $P=\left(F_{\mathrm{o}}^{2}+2 F_{\mathrm{c}}^{2}\right) / 3$

$(\Delta / \sigma)_{\max }<0.001$

$\Delta \rho_{\max }=0.16 \mathrm{e} \AA^{-3}$

$\Delta \rho_{\min }=-0.16$ e $\AA^{-3}$

Extinction correction: SHELXL2014 (Sheldrick, $2015 b), \mathrm{Fc}^{*}=\mathrm{kFc}\left[1+0.001 \mathrm{xFc}^{2} \lambda^{3} / \sin (2 \theta)\right]^{-1 / 4}$

Extinction coefficient: 0.0046 (8)

Absolute structure: Refined as an inversion twin

Absolute structure parameter: 0.2 (4) 


\section{Special details}

Geometry. All esds (except the esd in the dihedral angle between two 1.s. planes) are estimated using the full covariance matrix. The cell esds are taken into account individually in the estimation of esds in distances, angles and torsion angles; correlations between esds in cell parameters are only used when they are defined by crystal symmetry. An approximate (isotropic) treatment of cell esds is used for estimating esds involving l.s. planes.

Refinement. Refinement of $\mathrm{F}^{2}$ against ALL reflections. The weighted R-factor $\mathrm{wR}$ and goodness of fit $\mathrm{S}$ are based on $\mathrm{F}^{2}$, conventional $\mathrm{R}$-factors $\mathrm{R}$ are based on $\mathrm{F}$, with $\mathrm{F}$ set to zero for negative $\mathrm{F}^{2}$. The threshold expression of $\mathrm{F}^{2}>2 \operatorname{sigma}\left(\mathrm{F}^{2}\right)$ is used only for calculating R-factors(gt) etc. and is not relevant to the choice of reflections for refinement. R-factors based on $\mathrm{F}^{2}$ are statistically about twice as large as those based on F, and R- factors based on ALL data will be even larger.

Refined as a 2-component inversion twin.

Fractional atomic coordinates and isotropic or equivalent isotropic displacement parameters $\left(\AA^{2}\right)$

\begin{tabular}{|c|c|c|c|c|}
\hline & $x$ & $y$ & $z$ & $U_{\text {iso }} * / U_{\text {eq }}$ \\
\hline $\mathrm{O} 1$ & $0.5328(3)$ & $0.41857(14)$ & $0.9098(2)$ & $0.0351(5)$ \\
\hline N1 & $0.3365(4)$ & $0.19467(18)$ & $0.4307(3)$ & $0.0312(5)$ \\
\hline $\mathrm{N} 2$ & $0.6135(4)$ & $0.50398(17)$ & $0.7228(3)$ & $0.0296(5)$ \\
\hline $\mathrm{H} 2 \mathrm{~A}$ & $0.604(5)$ & $0.510(3)$ & $0.624(4)$ & $0.045(10)^{*}$ \\
\hline N3 & $0.6784(3)$ & $0.58400(17)$ & $0.8203(3)$ & $0.0296(5)$ \\
\hline $\mathrm{C} 1$ & $0.4132(4)$ & $0.1685(2)$ & $0.5821(3)$ & $0.0290(6)$ \\
\hline $\mathrm{C} 2$ & $0.4235(4)$ & $0.0645(2)$ & $0.6177(3)$ & $0.0344(7)$ \\
\hline $\mathrm{H} 2$ & 0.3728 & 0.0170 & 0.5384 & $0.041^{*}$ \\
\hline $\mathrm{C} 3$ & $0.5054(5)$ & $0.0318(2)$ & $0.7645(3)$ & $0.0361(7)$ \\
\hline H3 & 0.5112 & -0.0382 & 0.7874 & $0.043^{*}$ \\
\hline $\mathrm{C} 4$ & $0.5811(5)$ & $0.1021(2)$ & $0.8818(3)$ & $0.0360(7)$ \\
\hline $\mathrm{H} 4$ & 0.6419 & 0.0790 & 0.9832 & $0.043^{*}$ \\
\hline $\mathrm{C} 5$ & $0.5688(4)$ & $0.2030(2)$ & $0.8526(3)$ & $0.0331(7)$ \\
\hline H5 & 0.6186 & 0.2492 & 0.9343 & $0.040^{*}$ \\
\hline C6 & $0.4826(4)$ & $0.2396(2)$ & $0.7019(3)$ & $0.0282(6)$ \\
\hline $\mathrm{C} 7$ & $0.4633(4)$ & $0.3437(2)$ & $0.6587(3)$ & $0.0280(6)$ \\
\hline $\mathrm{C} 8$ & $0.3796(4)$ & $0.3683(2)$ & 0.5077 (3) & $0.0294(6)$ \\
\hline H8 & 0.3617 & 0.4370 & 0.4780 & $0.035^{*}$ \\
\hline C9 & $0.3195(4)$ & $0.2915(2)$ & $0.3955(3)$ & $0.0296(6)$ \\
\hline $\mathrm{C} 10$ & $0.2341(4)$ & $0.3167(2)$ & $0.2296(3)$ & $0.0318(7)$ \\
\hline $\mathrm{C} 11$ & $0.1402(5)$ & $0.4069(2)$ & $0.1854(4)$ & $0.0373(7)$ \\
\hline H11 & 0.1363 & 0.4552 & 0.2616 & $0.045^{*}$ \\
\hline $\mathrm{C} 12$ & $0.0520(5)$ & $0.4267(3)$ & $0.0301(4)$ & $0.0460(8)$ \\
\hline H12 & -0.0135 & 0.4877 & 0.0007 & $0.055^{*}$ \\
\hline $\mathrm{C} 13$ & $0.0603(5)$ & $0.3570(3)$ & $-0.0810(4)$ & $0.0479(9)$ \\
\hline H13 & -0.0009 & 0.3698 & -0.1868 & $0.058^{*}$ \\
\hline $\mathrm{C} 14$ & $0.1574(6)$ & $0.2689(3)$ & $-0.0383(4)$ & $0.0477(9)$ \\
\hline H14 & 0.1659 & 0.2224 & -0.1155 & $0.057^{*}$ \\
\hline $\mathrm{C} 15$ & $0.2429(5)$ & $0.2475(3)$ & $0.1164(4)$ & $0.0400(8)$ \\
\hline H15 & 0.3071 & 0.1859 & 0.1449 & $0.048^{*}$ \\
\hline $\mathrm{C} 16$ & $0.5387(4)$ & $0.4248(2)$ & $0.7759(3)$ & $0.0278(6)$ \\
\hline $\mathrm{C} 17$ & $0.7413(4)$ & $0.6576(2)$ & $0.7606(3)$ & $0.0293(6)$ \\
\hline H17 & 0.7424 & 0.6530 & 0.6563 & $0.035^{*}$ \\
\hline $\mathrm{C} 18$ & $0.8117(4)$ & $0.7491(2)$ & $0.8492(3)$ & $0.0278(6)$ \\
\hline
\end{tabular}




$\begin{array}{lllll}\mathrm{C} 19 & 0.8098(5) & 0.7608(2) & 1.0015(3) & 0.0349(7) \\ \mathrm{H} 19 & 0.7640 & 0.7083 & 1.0510 & 0.042^{*} \\ \mathrm{C} 20 & 0.8744(5) & 0.8485(2) & 1.0807(4) & 0.0381(7) \\ \mathrm{H} 20 & 0.8715 & 0.8565 & 1.1841 & 0.046^{*} \\ \mathrm{C} 21 & 0.9431(5) & 0.9247(2) & 1.0102(4) & 0.0381(7) \\ \mathrm{H} 21 & 0.9869 & 0.9850 & 1.0653 & 0.046^{*} \\ \mathrm{C} 22 & 0.9483(5) & 0.9136(2) & 0.8603(4) & 0.0371(7) \\ \mathrm{H} 22 & 0.9976 & 0.9655 & 0.8125 & 0.044^{*} \\ \mathrm{C} 23 & 0.8806(4) & 0.8257(2) & 0.7794(3) & 0.0328(7) \\ \mathrm{H} 23 & 0.8818 & 0.8184 & 0.6755 & 0.039^{*}\end{array}$

Atomic displacement parameters $\left(\AA^{2}\right)$

\begin{tabular}{|c|c|c|c|c|c|c|}
\hline & $U^{11}$ & $U^{22}$ & $U^{33}$ & $U^{12}$ & $U^{13}$ & $U^{23}$ \\
\hline $\mathrm{O} 1$ & $0.0532(14)$ & $0.0323(11)$ & $0.0202(10)$ & $0.0008(10)$ & $0.0114(9)$ & $-0.0002(8)$ \\
\hline N1 & $0.0352(15)$ & $0.0334(13)$ & $0.0238(12)$ & -0.0015 & $0.0065(10)$ & $0.0007(10)$ \\
\hline $\mathrm{N} 2$ & $0.0400(15)$ & $0.0293(12)$ & $0.0177(10)$ & $-0.0012(10)$ & $0.0058(9)$ & $-0.0007(9)$ \\
\hline N3 & $0.0354(15)$ & $0.0285(12)$ & $0.0223(11)$ & $-0.0006(10)$ & $0.0044(10)$ & $-0.0023(9)$ \\
\hline $\mathrm{C} 1$ & $0.0334(16)$ & $0.0288(14)$ & $0.0237(13)$ & $0.0004(12)$ & $0.0070(11)$ & $-0.0004(11)$ \\
\hline $\mathrm{C} 2$ & $0.0410(19)$ & $0.0311(15)$ & $0.0302(16)$ & $-0.0017(14)$ & $0.0093(14)$ & $-0.0037(12)$ \\
\hline $\mathrm{C} 3$ & $0.045(2)$ & $0.0270(15)$ & $0.0353(16)$ & $0.0014(13)$ & $0.0107(14)$ & $0.0043(12)$ \\
\hline $\mathrm{C} 4$ & $0.047(2)$ & $0.0327(16)$ & $0.0254(15)$ & $0.0025(14)$ & $0.0064(13)$ & $0.0031(11)$ \\
\hline $\mathrm{C} 5$ & $0.0429(19)$ & $0.0321(15)$ & $0.0218(14)$ & $0.0003(13)$ & $0.0058(13)$ & $0.0000(12)$ \\
\hline C6 & $0.0323(17)$ & $0.0297(15)$ & $0.0222(13)$ & $0.0016(12)$ & $0.0076(11)$ & $0.0011(11)$ \\
\hline $\mathrm{C} 7$ & $0.0326(17)$ & $0.0275(14)$ & $0.0233(13)$ & $0.0008(12)$ & $0.0075(11)$ & $-0.0006(11)$ \\
\hline $\mathrm{C} 8$ & $0.0361(17)$ & $0.0291(14)$ & $0.0213(13)$ & $0.0004(12)$ & $0.0057(11)$ & $0.0015(11)$ \\
\hline C9 & $0.0308(17)$ & $0.0338(15)$ & $0.0229(13)$ & $-0.0011(13)$ & $0.0059(12)$ & $-0.0002(11)$ \\
\hline $\mathrm{C} 10$ & $0.0309(17)$ & $0.0399(17)$ & $0.0219(14)$ & $-0.0039(13)$ & $0.0038(12)$ & $0.0002(12)$ \\
\hline $\mathrm{C} 11$ & $0.0402(19)$ & $0.0422(17)$ & $0.0254(15)$ & $0.0001(14)$ & $0.0034(12)$ & $0.0025(13)$ \\
\hline $\mathrm{C} 12$ & $0.044(2)$ & $0.052(2)$ & $0.0355(18)$ & $0.0004(16)$ & 0.0009 (14) & $0.0093(15)$ \\
\hline $\mathrm{C} 13$ & $0.049(2)$ & $0.065(2)$ & $0.0227(16)$ & $-0.0093(18)$ & $0.0005(14)$ & $0.0049(15)$ \\
\hline C14 & $0.054(2)$ & $0.061(2)$ & $0.0237(15)$ & $-0.0054(18)$ & $0.0058(14)$ & $-0.0045(15)$ \\
\hline $\mathrm{C} 15$ & $0.046(2)$ & $0.0445(18)$ & $0.0262(15)$ & $-0.0002(15)$ & $0.0060(13)$ & $-0.0019(13)$ \\
\hline $\mathrm{C} 16$ & $0.0352(17)$ & $0.0272(14)$ & $0.0192(14)$ & $0.0029(12)$ & $0.0049(11)$ & $0.0004(10)$ \\
\hline $\mathrm{C} 17$ & $0.0337(17)$ & $0.0315(15)$ & $0.0214(13)$ & $0.0016(12)$ & $0.0060(11)$ & $0.0018(11)$ \\
\hline $\mathrm{C} 18$ & $0.0279(16)$ & $0.0308(14)$ & $0.0220(13)$ & $0.0019(12)$ & $0.0033(11)$ & $0.0000(11)$ \\
\hline C19 & $0.0392(18)$ & $0.0399(17)$ & $0.0260(15)$ & $-0.0053(14)$ & $0.0103(13)$ & $-0.0014(12)$ \\
\hline $\mathrm{C} 20$ & $0.043(2)$ & $0.0463(18)$ & $0.0248(14)$ & $-0.0055(15)$ & $0.0104(13)$ & $-0.0066(13)$ \\
\hline $\mathrm{C} 21$ & $0.0415(19)$ & $0.0350(17)$ & $0.0349(17)$ & $-0.0007(14)$ & $0.0068(14)$ & $-0.0077(13)$ \\
\hline $\mathrm{C} 22$ & $0.0451(19)$ & $0.0302(15)$ & $0.0341(16)$ & $-0.0011(13)$ & $0.0090(13)$ & $0.0016(12)$ \\
\hline $\mathrm{C} 23$ & $0.0398(19)$ & $0.0330(15)$ & $0.0236(14)$ & $0.0033(13)$ & $0.0065(12)$ & $0.0022(11)$ \\
\hline
\end{tabular}

Geometric parameters $\left(\stackrel{A}{\circ}{ }^{\circ}\right)$

\begin{tabular}{llll}
\hline $\mathrm{O} 1-\mathrm{C} 16$ & $1.231(3)$ & $\mathrm{C} 10-\mathrm{C} 15$ & $1.395(4)$ \\
$\mathrm{N} 1-\mathrm{C} 9$ & $1.324(3)$ & $\mathrm{C} 11-\mathrm{C} 12$ & $1.394(4)$ \\
$\mathrm{N} 1-\mathrm{C} 1$ & $1.367(4)$ & $\mathrm{C} 11-\mathrm{H} 11$ & 0.9500 \\
$\mathrm{~N} 2-\mathrm{C} 16$ & $1.353(4)$ & $\mathrm{C} 12-\mathrm{C} 13$ & $1.382(5)$
\end{tabular}




\begin{tabular}{|c|c|c|c|}
\hline $\mathrm{N} 2-\mathrm{N} 3$ & $1.378(3)$ & $\mathrm{C} 12-\mathrm{H} 12$ & 0.9500 \\
\hline $\mathrm{N} 2-\mathrm{H} 2 \mathrm{~A}$ & $0.88(4)$ & $\mathrm{C} 13-\mathrm{C} 14$ & $1.381(5)$ \\
\hline N3-C17 & $1.279(4)$ & $\mathrm{C} 13-\mathrm{H} 13$ & 0.9500 \\
\hline $\mathrm{C} 1-\mathrm{C} 2$ & $1.416(4)$ & $\mathrm{C} 14-\mathrm{C} 15$ & $1.390(5)$ \\
\hline $\mathrm{C} 1-\mathrm{C} 6$ & $1.418(4)$ & $\mathrm{C} 14-\mathrm{H} 14$ & 0.9500 \\
\hline $\mathrm{C} 2-\mathrm{C} 3$ & $1.363(4)$ & $\mathrm{C} 15-\mathrm{H} 15$ & 0.9500 \\
\hline $\mathrm{C} 2-\mathrm{H} 2$ & 0.9500 & $\mathrm{C} 17-\mathrm{C} 18$ & $1.469(4)$ \\
\hline $\mathrm{C} 3-\mathrm{C} 4$ & $1.406(4)$ & $\mathrm{C} 17-\mathrm{H} 17$ & 0.9500 \\
\hline $\mathrm{C} 3-\mathrm{H} 3$ & 0.9500 & $\mathrm{C} 18-\mathrm{C} 23$ & $1.383(4)$ \\
\hline $\mathrm{C} 4-\mathrm{C} 5$ & $1.365(4)$ & $\mathrm{C} 18-\mathrm{C} 19$ & $1.393(4)$ \\
\hline $\mathrm{C} 4-\mathrm{H} 4$ & 0.9500 & $\mathrm{C} 19-\mathrm{C} 20$ & $1.382(4)$ \\
\hline $\mathrm{C} 5-\mathrm{C} 6$ & $1.416(4)$ & $\mathrm{C} 19-\mathrm{H} 19$ & 0.9500 \\
\hline $\mathrm{C} 5-\mathrm{H} 5$ & 0.9500 & $\mathrm{C} 20-\mathrm{C} 21$ & $1.383(5)$ \\
\hline $\mathrm{C} 6-\mathrm{C} 7$ & $1.434(4)$ & $\mathrm{C} 20-\mathrm{H} 20$ & 0.9500 \\
\hline $\mathrm{C} 7-\mathrm{C} 8$ & $1.367(4)$ & $\mathrm{C} 21-\mathrm{C} 22$ & $1.379(4)$ \\
\hline $\mathrm{C} 7-\mathrm{C} 16$ & $1.504(4)$ & $\mathrm{C} 21-\mathrm{H} 21$ & 0.9500 \\
\hline $\mathrm{C} 8-\mathrm{C} 9$ & $1.418(4)$ & $\mathrm{C} 22-\mathrm{C} 23$ & $1.395(4)$ \\
\hline $\mathrm{C} 8-\mathrm{H} 8$ & 0.9500 & $\mathrm{C} 22-\mathrm{H} 22$ & 0.9500 \\
\hline $\mathrm{C} 9-\mathrm{C} 10$ & $1.489(4)$ & $\mathrm{C} 23-\mathrm{H} 23$ & 0.9500 \\
\hline $\mathrm{C} 10-\mathrm{C} 11$ & $1.395(4)$ & & \\
\hline $\mathrm{C} 9-\mathrm{N} 1-\mathrm{C} 1$ & $118.1(2)$ & $\mathrm{C} 10-\mathrm{C} 11-\mathrm{H} 11$ & 119.8 \\
\hline $\mathrm{C} 16-\mathrm{N} 2-\mathrm{N} 3$ & $118.8(2)$ & $\mathrm{C} 13-\mathrm{C} 12-\mathrm{C} 11$ & $119.7(3)$ \\
\hline $\mathrm{C} 16-\mathrm{N} 2-\mathrm{H} 2 \mathrm{~A}$ & $121(2)$ & $\mathrm{C} 13-\mathrm{C} 12-\mathrm{H} 12$ & 120.1 \\
\hline $\mathrm{N} 3-\mathrm{N} 2-\mathrm{H} 2 \mathrm{~A}$ & $119(2)$ & $\mathrm{C} 11-\mathrm{C} 12-\mathrm{H} 12$ & 120.1 \\
\hline $\mathrm{C} 17-\mathrm{N} 3-\mathrm{N} 2$ & $115.4(2)$ & $\mathrm{C} 14-\mathrm{C} 13-\mathrm{C} 12$ & $120.1(3)$ \\
\hline $\mathrm{N} 1-\mathrm{C} 1-\mathrm{C} 2$ & $117.1(2)$ & $\mathrm{C} 14-\mathrm{C} 13-\mathrm{H} 13$ & 120.0 \\
\hline $\mathrm{N} 1-\mathrm{C} 1-\mathrm{C} 6$ & $123.4(2)$ & $\mathrm{C} 12-\mathrm{C} 13-\mathrm{H} 13$ & 120.0 \\
\hline $\mathrm{C} 2-\mathrm{C} 1-\mathrm{C} 6$ & $119.5(3)$ & $\mathrm{C} 13-\mathrm{C} 14-\mathrm{C} 15$ & $120.7(3)$ \\
\hline $\mathrm{C} 3-\mathrm{C} 2-\mathrm{C} 1$ & $120.9(3)$ & $\mathrm{C} 13-\mathrm{C} 14-\mathrm{H} 14$ & 119.7 \\
\hline $\mathrm{C} 3-\mathrm{C} 2-\mathrm{H} 2$ & 119.6 & $\mathrm{C} 15-\mathrm{C} 14-\mathrm{H} 14$ & 119.7 \\
\hline $\mathrm{C} 1-\mathrm{C} 2-\mathrm{H} 2$ & 119.6 & $\mathrm{C} 14-\mathrm{C} 15-\mathrm{C} 10$ & $119.7(3)$ \\
\hline $\mathrm{C} 2-\mathrm{C} 3-\mathrm{C} 4$ & $119.6(3)$ & $\mathrm{C} 14-\mathrm{C} 15-\mathrm{H} 15$ & 120.1 \\
\hline $\mathrm{C} 2-\mathrm{C} 3-\mathrm{H} 3$ & 120.2 & $\mathrm{C} 10-\mathrm{C} 15-\mathrm{H} 15$ & 120.1 \\
\hline $\mathrm{C} 4-\mathrm{C} 3-\mathrm{H} 3$ & 120.2 & $\mathrm{O} 1-\mathrm{C} 16-\mathrm{N} 2$ & $123.2(3)$ \\
\hline $\mathrm{C} 5-\mathrm{C} 4-\mathrm{C} 3$ & $121.2(3)$ & $\mathrm{O} 1-\mathrm{C} 16-\mathrm{C} 7$ & $122.0(3)$ \\
\hline $\mathrm{C} 5-\mathrm{C} 4-\mathrm{H} 4$ & 119.4 & $\mathrm{~N} 2-\mathrm{C} 16-\mathrm{C} 7$ & $114.9(2)$ \\
\hline $\mathrm{C} 3-\mathrm{C} 4-\mathrm{H} 4$ & 119.4 & $\mathrm{~N} 3-\mathrm{C} 17-\mathrm{C} 18$ & $121.7(2)$ \\
\hline $\mathrm{C} 4-\mathrm{C} 5-\mathrm{C} 6$ & $120.6(3)$ & $\mathrm{N} 3-\mathrm{C} 17-\mathrm{H} 17$ & 119.1 \\
\hline $\mathrm{C} 4-\mathrm{C} 5-\mathrm{H} 5$ & 119.7 & $\mathrm{C} 18-\mathrm{C} 17-\mathrm{H} 17$ & 119.1 \\
\hline $\mathrm{C} 6-\mathrm{C} 5-\mathrm{H} 5$ & 119.7 & $\mathrm{C} 23-\mathrm{C} 18-\mathrm{C} 19$ & $119.2(3)$ \\
\hline $\mathrm{C} 5-\mathrm{C} 6-\mathrm{C} 1$ & $118.2(2)$ & $\mathrm{C} 23-\mathrm{C} 18-\mathrm{C} 17$ & $119.2(2)$ \\
\hline $\mathrm{C} 5-\mathrm{C} 6-\mathrm{C} 7$ & $125.1(3)$ & $\mathrm{C} 19-\mathrm{C} 18-\mathrm{C} 17$ & $121.6(3)$ \\
\hline $\mathrm{C} 1-\mathrm{C} 6-\mathrm{C} 7$ & $116.6(2)$ & $\mathrm{C} 20-\mathrm{C} 19-\mathrm{C} 18$ & $120.1(3)$ \\
\hline $\mathrm{C} 8-\mathrm{C} 7-\mathrm{C} 6$ & $118.9(3)$ & $\mathrm{C} 20-\mathrm{C} 19-\mathrm{H} 19$ & 120.0 \\
\hline $\mathrm{C} 8-\mathrm{C} 7-\mathrm{C} 16$ & $120.2(3)$ & $\mathrm{C} 18-\mathrm{C} 19-\mathrm{H} 19$ & 120.0 \\
\hline $\mathrm{C} 6-\mathrm{C} 7-\mathrm{C} 16$ & $120.8(3)$ & $\mathrm{C} 19-\mathrm{C} 20-\mathrm{C} 21$ & $120.4(3)$ \\
\hline $\mathrm{C} 7-\mathrm{C} 8-\mathrm{C} 9$ & $120.2(3)$ & $\mathrm{C} 19-\mathrm{C} 20-\mathrm{H} 20$ & 119.8 \\
\hline
\end{tabular}




\begin{tabular}{|c|c|c|c|}
\hline $\mathrm{C} 7-\mathrm{C} 8-\mathrm{H} 8$ & 119.9 & $\mathrm{C} 21-\mathrm{C} 20-\mathrm{H} 20$ & 119.8 \\
\hline $\mathrm{C} 9-\mathrm{C} 8-\mathrm{H} 8$ & 119.9 & $\mathrm{C} 22-\mathrm{C} 21-\mathrm{C} 20$ & $120.2(3)$ \\
\hline $\mathrm{N} 1-\mathrm{C} 9-\mathrm{C} 8$ & $122.6(3)$ & $\mathrm{C} 22-\mathrm{C} 21-\mathrm{H} 21$ & 119.9 \\
\hline $\mathrm{N} 1-\mathrm{C} 9-\mathrm{C} 10$ & $116.4(2)$ & $\mathrm{C} 20-\mathrm{C} 21-\mathrm{H} 21$ & 119.9 \\
\hline $\mathrm{C} 8-\mathrm{C} 9-\mathrm{C} 10$ & $121.0(3)$ & $\mathrm{C} 21-\mathrm{C} 22-\mathrm{C} 23$ & $119.5(3)$ \\
\hline $\mathrm{C} 11-\mathrm{C} 10-\mathrm{C} 15$ & $119.3(3)$ & $\mathrm{C} 21-\mathrm{C} 22-\mathrm{H} 22$ & 120.2 \\
\hline $\mathrm{C} 11-\mathrm{C} 10-\mathrm{C} 9$ & $121.2(3)$ & $\mathrm{C} 23-\mathrm{C} 22-\mathrm{H} 22$ & 120.2 \\
\hline $\mathrm{C} 15-\mathrm{C} 10-\mathrm{C} 9$ & $119.5(3)$ & $\mathrm{C} 18-\mathrm{C} 23-\mathrm{C} 22$ & $120.6(3)$ \\
\hline $\mathrm{C} 12-\mathrm{C} 11-\mathrm{C} 10$ & $120.4(3)$ & $\mathrm{C} 18-\mathrm{C} 23-\mathrm{H} 23$ & 119.7 \\
\hline $\mathrm{C} 12-\mathrm{C} 11-\mathrm{H} 11$ & 119.8 & $\mathrm{C} 22-\mathrm{C} 23-\mathrm{H} 23$ & 119.7 \\
\hline $\mathrm{C} 16-\mathrm{N} 2-\mathrm{N} 3-\mathrm{C} 17$ & $-177.6(3)$ & $\mathrm{C} 8-\mathrm{C} 9-\mathrm{C} 10-\mathrm{C} 15$ & $154.4(3)$ \\
\hline $\mathrm{C} 9-\mathrm{N} 1-\mathrm{C} 1-\mathrm{C} 2$ & $177.6(3)$ & $\mathrm{C} 15-\mathrm{C} 10-\mathrm{C} 11-\mathrm{C} 12$ & $1.6(5)$ \\
\hline $\mathrm{C} 9-\mathrm{N} 1-\mathrm{C} 1-\mathrm{C} 6$ & $-2.9(4)$ & $\mathrm{C} 9-\mathrm{C} 10-\mathrm{C} 11-\mathrm{C} 12$ & $-176.2(3)$ \\
\hline $\mathrm{N} 1-\mathrm{C} 1-\mathrm{C} 2-\mathrm{C} 3$ & $177.0(3)$ & $\mathrm{C} 10-\mathrm{C} 11-\mathrm{C} 12-\mathrm{C} 13$ & $-1.0(5)$ \\
\hline $\mathrm{C} 6-\mathrm{C} 1-\mathrm{C} 2-\mathrm{C} 3$ & $-2.5(5)$ & $\mathrm{C} 11-\mathrm{C} 12-\mathrm{C} 13-\mathrm{C} 14$ & $-0.8(6)$ \\
\hline $\mathrm{C} 1-\mathrm{C} 2-\mathrm{C} 3-\mathrm{C} 4$ & $-0.2(5)$ & $\mathrm{C} 12-\mathrm{C} 13-\mathrm{C} 14-\mathrm{C} 15$ & $2.1(6)$ \\
\hline $\mathrm{C} 2-\mathrm{C} 3-\mathrm{C} 4-\mathrm{C} 5$ & $2.2(5)$ & $\mathrm{C} 13-\mathrm{C} 14-\mathrm{C} 15-\mathrm{C} 10$ & $-1.5(5)$ \\
\hline $\mathrm{C} 3-\mathrm{C} 4-\mathrm{C} 5-\mathrm{C} 6$ & $-1.4(5)$ & $\mathrm{C} 11-\mathrm{C} 10-\mathrm{C} 15-\mathrm{C} 14$ & $-0.4(5)$ \\
\hline $\mathrm{C} 4-\mathrm{C} 5-\mathrm{C} 6-\mathrm{C} 1$ & $-1.4(4)$ & $\mathrm{C} 9-\mathrm{C} 10-\mathrm{C} 15-\mathrm{C} 14$ & $177.5(3)$ \\
\hline $\mathrm{C} 4-\mathrm{C} 5-\mathrm{C} 6-\mathrm{C} 7$ & $-179.6(3)$ & $\mathrm{N} 3-\mathrm{N} 2-\mathrm{C} 16-\mathrm{O} 1$ & $-3.3(4)$ \\
\hline $\mathrm{N} 1-\mathrm{C} 1-\mathrm{C} 6-\mathrm{C} 5$ & $-176.2(3)$ & $\mathrm{N} 3-\mathrm{N} 2-\mathrm{C} 16-\mathrm{C} 7$ & $177.0(3)$ \\
\hline $\mathrm{C} 2-\mathrm{C} 1-\mathrm{C} 6-\mathrm{C} 5$ & $3.3(4)$ & $\mathrm{C} 8-\mathrm{C} 7-\mathrm{C} 16-\mathrm{O} 1$ & $144.0(3)$ \\
\hline $\mathrm{N} 1-\mathrm{C} 1-\mathrm{C} 6-\mathrm{C} 7$ & $2.2(4)$ & $\mathrm{C} 6-\mathrm{C} 7-\mathrm{C} 16-\mathrm{O} 1$ & $-37.9(4)$ \\
\hline $\mathrm{C} 2-\mathrm{C} 1-\mathrm{C} 6-\mathrm{C} 7$ & $-178.3(3)$ & $\mathrm{C} 8-\mathrm{C} 7-\mathrm{C} 16-\mathrm{N} 2$ & $-36.4(4)$ \\
\hline $\mathrm{C} 5-\mathrm{C} 6-\mathrm{C} 7-\mathrm{C} 8$ & $178.8(3)$ & $\mathrm{C} 6-\mathrm{C} 7-\mathrm{C} 16-\mathrm{N} 2$ & $141.7(3)$ \\
\hline $\mathrm{C} 1-\mathrm{C} 6-\mathrm{C} 7-\mathrm{C} 8$ & $0.5(4)$ & $\mathrm{N} 2-\mathrm{N} 3-\mathrm{C} 17-\mathrm{C} 18$ & $178.7(3)$ \\
\hline $\mathrm{C} 5-\mathrm{C} 6-\mathrm{C} 7-\mathrm{C} 16$ & $0.6(4)$ & $\mathrm{N} 3-\mathrm{C} 17-\mathrm{C} 18-\mathrm{C} 23$ & $179.0(3)$ \\
\hline $\mathrm{C} 1-\mathrm{C} 6-\mathrm{C} 7-\mathrm{C} 16$ & $-177.6(3)$ & N3-C17-C18-C19 & $-1.3(5)$ \\
\hline $\mathrm{C} 6-\mathrm{C} 7-\mathrm{C} 8-\mathrm{C} 9$ & $-2.4(4)$ & $\mathrm{C} 23-\mathrm{C} 18-\mathrm{C} 19-\mathrm{C} 20$ & $0.7(5)$ \\
\hline $\mathrm{C} 16-\mathrm{C} 7-\mathrm{C} 8-\mathrm{C} 9$ & $175.8(3)$ & $\mathrm{C} 17-\mathrm{C} 18-\mathrm{C} 19-\mathrm{C} 20$ & $-179.0(3)$ \\
\hline $\mathrm{C} 1-\mathrm{N} 1-\mathrm{C} 9-\mathrm{C} 8$ & $0.9(4)$ & $\mathrm{C} 18-\mathrm{C} 19-\mathrm{C} 20-\mathrm{C} 21$ & $-0.7(5)$ \\
\hline $\mathrm{C} 1-\mathrm{N} 1-\mathrm{C} 9-\mathrm{C} 10$ & $-179.6(3)$ & $\mathrm{C} 19-\mathrm{C} 20-\mathrm{C} 21-\mathrm{C} 22$ & $-0.2(5)$ \\
\hline $\mathrm{C} 7-\mathrm{C} 8-\mathrm{C} 9-\mathrm{N} 1$ & $1.8(5)$ & $\mathrm{C} 20-\mathrm{C} 21-\mathrm{C} 22-\mathrm{C} 23$ & $1.2(5)$ \\
\hline $\mathrm{C} 7-\mathrm{C} 8-\mathrm{C} 9-\mathrm{C} 10$ & $-177.8(3)$ & $\mathrm{C} 19-\mathrm{C} 18-\mathrm{C} 23-\mathrm{C} 22$ & $0.3(5)$ \\
\hline $\mathrm{N} 1-\mathrm{C} 9-\mathrm{C} 10-\mathrm{C} 11$ & $152.7(3)$ & $\mathrm{C} 17-\mathrm{C} 18-\mathrm{C} 23-\mathrm{C} 22$ & $179.9(3)$ \\
\hline $\mathrm{C} 8-\mathrm{C} 9-\mathrm{C} 10-\mathrm{C} 11$ & $-27.8(4)$ & $\mathrm{C} 21-\mathrm{C} 22-\mathrm{C} 23-\mathrm{C} 18$ & $-1.2(5)$ \\
\hline $\mathrm{N} 1-\mathrm{C} 9-\mathrm{C} 10-\mathrm{C} 15$ & $-25.2(4)$ & & \\
\hline
\end{tabular}

Hydrogen-bond geometry $\left(A,{ }^{\circ}\right)$

\begin{tabular}{lllll}
\hline$D-\mathrm{H} \cdots A$ & $D-\mathrm{H}$ & $\mathrm{H} \cdots A$ & $D \cdots A$ & $D-\mathrm{H} \cdots A$ \\
\hline $\mathrm{C} 5-\mathrm{H} 5 \cdots \mathrm{O} 1$ & 0.95 & 2.34 & $2.939(3)$ & 121 \\
$\mathrm{~N} 2-\mathrm{H} 2 A \cdots \mathrm{O} 1^{\mathrm{i}}$ & $0.88(4)$ & $2.08(4)$ & $2.907(3)$ & $155(4)$ \\
$\mathrm{C} 8-\mathrm{H} 8 \cdots \mathrm{O} 1^{\mathrm{i}}$ & 0.95 & 2.50 & $3.288(3)$ & 140 \\
$\mathrm{C} 17-\mathrm{H} 17 \cdots \mathrm{O} 1^{\mathrm{i}}$ & 0.95 & 2.52 & $3.268(3)$ & 136 \\
\hline
\end{tabular}

Symmetry code: (i) $x,-y+1, z-1 / 2$. 